\title{
Dual antiplatelet therapy in the contemporary drug-eluting stents era: from vulnerable stents to vulnerable patients
}

\author{
Stefano Benenati ${ }^{1}$ (D) Italo Porto ${ }^{1,2}$ (iD) \\ Accepted: 5 February 2022 / Published online: 2 March 2022 \\ (c) The Author(s), under exclusive licence to Springer Science+Business Media, LLC, part of Springer Nature 2022
}

Keywords Dual antiplatelet therapy · Drug-eluting stent · Biodegradable polymer - Durable polymer Percutaneous coronary intervention

In a seminal subanalysis of the Patient Related OuTcomes with Endeavor vs. Cypher stenting Trial (PROTECT) trial published in 2014, Camenzind and colleagues concluded that "the incidence of stent thrombosis [ST] in DES [drugeluting stent] trials should not be evaluated independently of DAPT [dual antiplatelet therapy] use, and the optimal duration of DAPT will likely depend upon stent type" [1]. It was, at the time, the most elegant demonstration that the long-term efficacy of coronary revascularization with DES could not be untwined from an optimal (e.g., durable) antiplatelet therapy. Since then, several technical innovations have helped cutting the risk of stent-related events, optimizing the bleeding-ischemia trade-off [2]. Among them, the introduction of biodegradable polymer (BP) (and, thereafter, of polymer-free) DES promised a major paradigm shift, due to the link between polymer-induced inflammation and late ST [2]. In this line of reasoning, early disappearance or wholesale avoidance of the "plastic" drug reservoir would mean taking the advantages of DES in the early phase and avoid their late drawbacks, potentially reducing the need for extended DAPT.

Dr. Stefano Benenati MD

stefanobenenatimd@gmail.com

1 Cardiovascular Disease Chair, Department of Internal Medicine (Di.M.I.), University of Genoa, Largo Rosanna Benzi 10, 16132 Genoa, GE, Italy

2 Cardiovascular Disease Unit, IRCCS Ospedale Policlinico San Martino, IRCCS Italian Cardiology Network, Genova, Italy
In this issue of the Journal of Thrombosis and Thrombol$y$ sis, Dr. Chong and colleagues present a Bayesian network meta-analysis of 15 randomized controlled trials (RCTs) and 43,875 patients in which different DAPT durations, e.g. short (1-3 months), intermediate (4-6 months) and standard (7-12 months), were stratified according to the two polymer categories, namely durable polymer (DP) or BP [3]. Whereas no differences were noticed across or within DAPT regimens regarding ischemic endpoints, including cardiac death, myocardial infarction (MI), target vessel revascularization, stroke and ST, BP-DES were surprisingly associated with a higher incidence of major bleeding. Indeed, not only the "standard DAPT" length of 7-12 months, but even the "intermediate" (4-6 months) and "short" (1-3 months) DAPT regimens, coupled with BP-DES, were associated with more bleeding as compared to short DAPT in the DP arm. Short DAPT with DP-DES (short-DP), noticeably, carried a more-than-halved risk of major bleeding compared to same-length DAPT with BP-DES (short-BP). Contrariwise, when examining the DP-DES results, only standard DAPT increased the risk of bleeding, whereas intermediate DAPT did not.

Starting from highly complex statistical fundamentals, network meta-analyses allow synthetizing direct and indirect data to obtain multiple treatments comparisons. As such, they potentially fill important gaps in the knowledge and generate hypotheses for future research. Paradoxically, indirect comparisons might be considered of the highest interest, as, by definition, thay have not been previously investigated in head-to-head studies. On the other hand, conclusions exclusively derived by indirect comparisons 
should be cautiously interpreted. This might be the case for the work by Dr. Chon and colleagues, in which various comparisons, including that between short-BP and short-DP and that between the latter strategy and intermediate DAPT with BP-DES, are entirely built in the absence of direct data.

While the increased probability of major bleeding with standard DAPT, as compared to the short one, was already reported $[4,5]$, the striking advantage of the short-DP group compared to the short-BP one is, at first sight, surprising. As the Authors themselves acknowledge, this finding is hardly explainable on the base of the sole stent design and likely influenced by the differences between the RCTs included in each arm. Specifically, it is not difficult to notice that the "driving force" of the BP-based regimens, especially for the short-BP arm, is the GLOBAL LEADERS trial [6], currently the largest RCT comparing a short DAPT regimen (1 month) with a standard one. According to the trial design, patients in the experimental arm received aspirin and ticagrelor for 1 month followed by ticagrelor monotherapy for 23 months, irrespective of clinical presentation, whereas the control arm was treated with a 12-month association of aspirin and ticagrelor (in patients with acute coronary syndromes, ACS) or clopidogrel (in those with chronic coronary syndromes), followed by aspirin monotherapy. The study failed to prove the superiority of the experimental treatment for the composite outcome of death and new Q-wave MI, showing substantial equipoise between the two arms for all considered bleeding endpoints. Several hypotheses have been put forward to explain the study findings regarding the latter outcomes. These include the blunted benefit of the experimental strategy in chronic patients, in whom the comparator was the association of aspirin and clopidogrel, rather than aspirin and ticagrelor; the confounding effect of the second year of treatment, during which the experimental arm (ticagrelor $90 \mathrm{mg}$ twice a day) was in fact compared with aspirin monotherapy rather than with DAPT itself, partially dissipating the beneficial effect obtained during the first year [7]; the open-label design and the absence of an external adjudication committee.

All together, these aspects might have "neutralized" the differences between short-BP and standard-length DAPT coupled with BP-DES, potentially explaining the comparable advantage of short-DP over both those strategies with regard to bleeding.

As elegantly proven by Chong et al. [3] and already shown by other studies [8], in the "modern DES" era, the demonstration of large efficacy divergences between different stent platforms, even across increasingly short DAPT regimens, is far-fetched. As devices become safer and safer, cardiologists should probably try to tailor DAPT duration according to a comprehensive evaluation of patient's vulnerability (intended as the proneness to both ischemic and bleeding events), rather than to the features of each specific device [9].

Supplementary Information The online version contains supplementary material available at https://doi.org/10.1007/s11239022-02639-z.

Acknowledgements none.

Conflict of interest Dr. Benenati has no conflicts of interest to disclose. Prof. Porto reports grants and personal fees from AstraZeneca, personal fees from Daiichi Sankyo, personal fees from Terumo Corporation, personal fees from Biotronik, personal fees from Bayer, personal fees from Amgen, personal fees and non-financial support from Abiomed, outside the present work.

\section{References}

1. Camenzind E, Boersma E, Wijns W et al (2014) Modifying effect of dual antiplatelet therapy on incidence of stent thrombosis according to implanted drug-eluting stent type. Eur Heart J Aug 1(29):1932-1948. https://doi.org/10.1093/eurheartj/ehu084

2. Torii S, Jinnouchi H, Sakamoto A et al (2020) Drug-eluting coronary stents: insights from preclinical and pathology studies. Nat Rev Cardiol Jan 17(1):37-51. https://doi.org/10.1038/ s41569-019-0234-x

3. Chong B, Goh RSJ, Kong G et al (2022) Comparison of biodegradable and newer generation durable polymer drug-eluting stents with short-term dual antiplatelet therapy: a systematic review and Bayesian network meta-analysis of randomized trials comprising of 43,875 patients. J Thromb Thrombolysis Jan 4. https://doi.org/10.1007/s11239-021-02628-8

4. Benenati S, Crimi G, Canale C et al (2020) Duration of dual antiplatelet therapy and subsequent monotherapy type in patients undergoing drug eluting stent implantation: a network Metaanalysis. Eur Heart J Cardiovasc Pharmacother Nov 2. https:// doi.org/10.1093/ehjcvp/pvaa127

5. Benenati S, Galli M, De Marzo V et al (2021) Very short vs. long dual antiplatelet therapy after second generation drug-eluting stents in 35785 patients undergoing percutaneous coronary interventions: a meta-analysis of randomized controlled trials. Eur Heart J Cardiovasc Pharmacother Mar 15(2):86-93. https://doi. org/10.1093/ehjcvp/pvaa001

6. Vranckx P, Valgimigli M, Juni P et al (2018) Ticagrelor plus aspirin for 1 month, followed by ticagrelor monotherapy for 23 months vs aspirin plus clopidogrel or ticagrelor for 12 months, followed by aspirin monotherapy for 12 months after implantation of a drug-eluting stent: a multicentre, open-label, randomised superiority trial. Lancet Sep 15(10151):940-949. https://doi. org/10.1016/S0140-6736(18)31858-0

7. Tomaniak M, Chichareon P, Onuma Y et al (2019) Benefit and Risks of Aspirin in Addition to Ticagrelor in Acute Coronary Syndromes: A Post Hoc Analysis of the Randomized GLOBAL LEADERS Trial. JAMA Cardiol Nov 1(11):1092-1101. https:// doi.org/10.1001/jamacardio.2019.3355

8. Dangas G, Baber U, Sharma SK et al (2021) Safety and Efficacy of Ticagrelor Monotherapy According to Drug-Eluting Stent Type: The TWILIGHT-STENT Study. EuroIntervention Aug 26. https://doi.org/10.4244/EIJ-D-21-00721

9. Galli M, Angiolillo DJ (2021) Antiplatelet therapy in percutaneous coronary intervention: latest evidence from randomized 
controlled trials. Curr Opin Cardiol Jul 1(4):390-396. https://doi.
org/10.1097/HCO.0000000000000879

Publisher's Note Springer Nature remains neutral with regard to jurisdictional claims in published maps and institutional affiliations. 